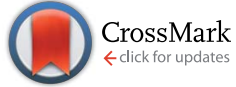

Cite this: RSC Adv., 2016, 6, 56544

\title{
Synthesis of imidazolium-crosslinked chitosan aerogel and its prospect as a dye removing adsorbent $\uparrow$
}

\author{
Juho Antti Sirviö, ${ }^{*}$ Miikka Visanko and Henrikki Liimatainen \\ The potential utility of Debus-Radziszewski imidazole synthesis in the fabrication of crosslinked chitosan was \\ studied. Three-component crosslinking was achieved by using glyoxal and propionaldehyde to connect amine \\ groups of chitosan via imidazolium crosslinking. A water-insoluble (at $\mathrm{pH}$ range of 2-10) chitosan was \\ obtained at room temperature with a degree of substitution of 0.45 and aerogel was obtained after freeze- \\ drying. The ability of the imidazolium-crosslinked chitosan (ICC) aerogel to absorb an anionic dye, Direct \\ Yellow 27, from a model water was then studied. Based on the Langmuir isotherm, at a $\mathrm{pH}$ of 4 , an \\ adsorption maximum of $2340 \mathrm{mg} \mathrm{g}^{-1}\left(3.5 \mathrm{mmol} \mathrm{g}^{-1}\right)$ was obtained. In addition, due to the permanent \\ cationic charge of imidazolium group, ICC exhibited excellent adsorption capacity, even under alkaline \\ conditions. Methylglyoxal and benzaldehyde were also used to obtain other types of ICC, demonstrating \\ the versatility of Debus-Radziszewski imidazole synthesis for fabrication of modified chitosan.
}

Received 31st March 2016

Accepted 7th June 2016

DOI: $10.1039 / \mathrm{c} 6 \mathrm{ra0} 8301 \mathrm{c}$

www.rsc.org/advances

\section{Introduction}

Chitosan is a semi-synthetic polymer obtained from naturally occurring chitin (the second most abundant polysaccharide after cellulose ${ }^{1}$ ) following alkaline hydrolysis (deacetylation). ${ }^{2,3}$ Chitosan is superior to chitin in a number of ways. For example, it has an abundance of reactive primary amino groups and solubility in water at a low $\mathrm{pH}$. Due to the cationic nature of its amino groups, chitosan has been used in various applications for ion exchange and dye removal. In addition, its biocompatibility, biodegradability, and low toxicity mean that chitosan can be employed in many medical applications. ${ }^{4-7}$

Chitosan can function as a solid absorbent of toxic and harmful dyes and of anions and heavy metals from waste waters. ${ }^{8-11}$ However, due to its high solubility under acidic solutions, chemical modification of chitosan is necessary to reduce its solubility and improve the recyclability of the solid adsorbent. ${ }^{12}$ The absorption capacity and recyclability of chitosan have been improved by grafting polymer side-chains onto chitosan..$^{12}$ However, grafting chitosan with oil-based macromolecules decreases the bio-content of the absorbent and may induce problems related to unreacted toxic monomers. Acidinsoluble chitosan can be produced by crosslinking of chitosan chains with various reagents, such as epichlorohydrin and

Fiber and Particle Engineering Research Unit, University of Oulu, P.O. Box 4300, FI-90014, Finland. E-mail: juho.sirvio@oulu.fi

$\dagger$ Electronic supplementary information (ESI) available: Chemical structure of Direct Yellow 27; FESEM images of ICC1 aerogel; schematic illustration of the synthesis of imidazolium crosslinked chitosan using methylglyoxal and benzaldehyde. See DOI: 10.1039/c6ra08301c glutaraldehyde. However, these chemicals possess a number of disadvantages, such as toxicity. ${ }^{13}$ Chemical modifications can also decrease the cationicity (e.g. the conversion of amine groups to corresponding imines by glutaraldehyde) of chitosan, resulting in a decline in its absorption capacity. ${ }^{13}$

Debus-Radziszewski imidazole synthesis is a threecomponent chemical reaction where two amino groups react with vicinal dicarbonyl and carbonyl reagents to form a stable imidazole structure. ${ }^{14}$ The requirement of only an acid catalyst makes Debus-Radziszewski imidazole synthesis an environmentally friendly reaction, as water is the only by-product. Recently, this reaction was efficiently used to crosslink poly(L-lysine) ${ }^{15}$ The Debus-Radziszewski imidazole synthesis allows the alteration of reaction products by the varying of each components (amine, carbonyl and dicarbonyl), and consequently the reaction has been used to obtain various imidazole-based ionic liquids. ${ }^{16}$ The fact that dicarbonyl and carbonyl components can be obtained from natural resources enhances the sustainability of Debus-Radziszewski imidazole synthesis.

In this study, Debus-Radziszewski imidazole synthesis was used to obtain water-insoluble chitosan using glyoxal and propionaldehyde as dicarbonyl and carbonyl components, respectively. Crosslinked chitosan was characterized using elemental analysis and diffusion reflectance Fourier transform infrared spectroscopy and its performance as an anion exchange material was tested by examining the adsorption of an anionic dye from a model water solution. The effect of the solution $\mathrm{pH}$, contact time, and initial dye concentration on the adsorption were studied. The versatility of Debus-Radziszewski imidazole synthesis in chitosan crosslinking was demonstrated using 
methylglyoxal and benzaldehyde as alternative dicarbonyl and carbonyl components, respectively.

\section{Materials and methods}

Chitosan (medium molecular weight), propionaldehyde, glyoxal, methylglyoxal, and benzaldehyde were obtained from Sigma Aldrich (Germany) and were used without further purifications. Acetic acid and $0.1 \mathrm{M}$ hydrochloride acid were purchased from Fluka (Germany). The acetic acid was diluted with deionized water to obtain a $10 \%$ solution. Direct Yellow 27 for the adsorption experiments was obtained from Sigma Aldrich (Germany). Deionized water was used throughout the experiments.

\section{Crosslinking of chitosan}

One gram (6.1 mmol) of chitosan was first dissolved in $100 \mathrm{~mL}$ of $10 \%$ acetic acid and then mixed with $0.35 \mathrm{~mL}(3.1 \mathrm{mmol})$ of glyoxal $(40 \%$ in water) and $0.22 \mathrm{~mL}(3.1 \mathrm{mmol})$ of propionaldehyde. The reaction was started by adjusting the $\mathrm{pH}$ to 5 by adding $10 \% \mathrm{NaOH}$ solution under vigorous stirring. The reaction was allowed to proceed for 2 days at room temperature. The chitosan gel that formed was transferred to a beaker containing $600 \mathrm{~mL}$ of water and mixed for $1 \mathrm{~h}$, after which mixture was filtrated and the product washed with $2 \mathrm{~L}$ of water. The obtained hydrogel was then freeze-dried to obtain an imidazolecrosslinked chitosan (ICC1) aerogel.

A similar procedure was employed to obtain ICC2 and ICC3, using $0.35 \mathrm{~mL}(3.1 \mathrm{mmol})$ of glyoxal $(40 \%$ in water) and 3.1 mmol of benzaldehyde and $0.497 \mathrm{~mL}(3.1 \mathrm{mmol})$ of methylglyoxal ( $40 \%$ in water) and $0.22 \mathrm{~mL}(3.1 \mathrm{mmol})$ of propionaldehyde, respectively. These products (ICC2 and ICC3) were only used to demonstrate the feasibility of the synthesis for alternative reactants.

The degree of substitution was calculated according to eqn (1) (ref. 17) after elemental analysis (PerkinElmer CHNS/O 2400 Series II, USA).

$$
\mathrm{DS}=\frac{\left(\frac{\mathrm{C}}{\mathrm{N}}\right)_{m}-\left(\frac{\mathrm{C}}{\mathrm{N}}\right)_{0}}{n}
$$

where $(\mathrm{C} / \mathrm{N})_{m}$ is the carbon-nitrogen ratio of the chitosan derivate, $(\mathrm{C} / \mathrm{N})_{0}$ is the carbon-nitrogen ratio of chitosan, and $n$ is the number of the carbon introduced during the chitosan derivatization. Carbon and nitrogen contents of original chitosan, ICC1, ICC2, and ICC3 are presented in Table 1.

In addition, the deacetylation degree $\left(X_{\mathrm{D}}\right)$ of the original chitosan was calculated using eqn (2): ${ }^{18}$

$$
X_{\mathrm{D}}=100 \times\left(4-0.583093 \times w_{\mathrm{C} / \mathrm{N}}\right)
$$

where $w_{\mathrm{C} / \mathrm{N}}$ is the carbon-nitrogen ratio of chitosan. $X_{\mathrm{D}}$ of original chitosan was calculated to be $67.2 \%$.

\section{Diffusion reflectance Fourier transform infrared spectroscopy}

Diffuse reflectance infrared Fourier transform (DRIFT) spectroscopy was used to characterize the ICC. The spectra of
Table 1 Carbon and nitrogen contents of original and crosslinked chitosan's determined by elemental analysis

\begin{tabular}{lll}
\hline Sample & Carbon (\%) & Nitrogen (\%) \\
\hline Chitosan & 41.3 & 7.23 \\
ICC1 & 39.0 & 5.53 \\
ICC2 & 39.2 & 3.33 \\
ICC3 & 34.4 & 2.84
\end{tabular}

chitosan and freeze-dried ICC were collected with a Bruker Vertex $80 \mathrm{v}$ spectrometer (USA). The spectra were obtained at a range of $600-4000 \mathrm{~cm}^{-1}$. For each sample, 40 scans were taken at a resolution of $2 \mathrm{~cm}^{-1}$.

\section{Field-emission scanning electron microscopy}

Field-Emission Scanning Electron Microscopy (FESEM) image of the ICC1 was obtained using Sigma HD VP FESEM (Zeiss, Germany). Sample was placed on carbon tape and sputtercoating with platinum (Pt). The accelerating voltage during imaging was $3 \mathrm{kV}$.

\section{Adsorption of anionic dye}

Adsorption experiments were carried out in batches. Dye stock solution of $1 \mathrm{~g} \mathrm{~L}^{-1}$ was prepared by dissolving the anionic dye (Direct Yellow 27) in deionized water, and dilutions of desired concentrations were made from the stock. $\mathrm{NaOH}$ and $\mathrm{HCl}$ were used to adjust the $\mathrm{pH}$ of the anionic dye solutions to 2-10. After dilution and $\mathrm{pH}$ adjustment, $100 \mathrm{mg} \mathrm{L}^{-1}$ of ICC were added, and the solution was shaken for $5 \mathrm{~min}$ to $24 \mathrm{~h}$ using a Flask Shaker (GWP, UK). Then, approximately $10 \mathrm{~mL}$ of the solution were collected with a syringe and filtrated using a $0.45 \mu \mathrm{m}$ cellulose acetate membrane (VWR, USA). The absorbance of the sample was measured spectrophotometrically at $393 \mathrm{~nm}$ using Shimadzu UV-Vis spectroscopy (Japan). The concentration of the dye in the sample and the quantity of the adsorbed dye were calculated using a calibration curve based on the absorbance results obtained with different dye solutions at different concentrations.

\section{Adsorption kinetics}

Pseudo-first-order and pseudo-second-order kinetics were employed to describe the kinetics of the adsorption of the dye by ICC1. The pseudo-first-order kinetic model is shown in eqn $(3),,^{19,20}$

$$
\frac{t}{q_{t}}=\frac{k_{1}}{q_{\mathrm{e}} t}+\frac{1}{q_{\mathrm{e}}}
$$

where $k_{1}(1 / \mathrm{min})$ is the rate constant of the pseudo-first-order adsorption, and $q_{t}$ and $q_{\mathrm{e}}$ are the anionic dye amounts adsorbed at time $t(\mathrm{~min})$ and equilibrium, respectively. The pseudosecond-order kinetic model is shown in eqn (4), ${ }^{21}$

$$
\frac{t}{q_{t}}=\frac{1}{k_{2} q_{\mathrm{e}}{ }^{2}}+\left(\frac{1}{q_{\mathrm{e}}}\right) t
$$


where $k_{2}\left(\mathrm{~g} \mathrm{mmol}^{-1} \mathrm{~min}^{-1}\right)$ is the rate constant of the pseudosecond-order adsorption.

\section{Adsorption isotherms}

The adsorption mechanisms were studied using Langmuir and Freundlich isotherm models. A linear form of the Langmuir isotherm is shown in eqn (6), ${ }^{20,22}$

$$
\frac{C_{\mathrm{e}}}{q_{\mathrm{e}}}=\left(\frac{1}{b Q_{0}}\right)+\left(\frac{C_{\mathrm{e}}}{Q_{0}}\right)
$$

where $C_{\mathrm{e}}$ is the equilibrium concentration of the adsorbate in the solution ( $\left.\mathrm{mmol} \mathrm{L}^{-1}\right), q_{\mathrm{e}}$ is the adsorbed amount $\left(\mathrm{mg} \mathrm{g}^{-1}\right)$ at equilibrium, $b\left(\mathrm{l} \mathrm{mmol}^{-1}\right)$ is a Langmuir constant that is related to the affinity of the binding sites, and $Q_{0}$ is the maximum adsorption capacity for monolayer formation on the adsorbent.

The logarithmic form of the Freundlich isotherm is shown in eqn (6), ${ }^{20}$

$$
\log q_{\mathrm{e}}=\log K_{\mathrm{F}}+\frac{1}{n}\left(\log C_{\mathrm{e}}\right)
$$

where $K_{\mathrm{F}}\left(\mathrm{mg}^{1-n} \mathrm{~g}^{-1} \mathrm{~L}^{-n}\right)$ and $n$ (dimensionless) represent the Freundlich constants.

\section{Results and discussion}

Debus-Radziszewski imidazole crosslinking of chitosan was performed in a $10 \%$ acetic acid solution using a stoichiometric amount ( $1 \mathrm{~mol}$ of carbonyl components per $2 \mathrm{~mol}$ of chitosan) of glyoxal and propionaldehyde at room temperature. Without adjusting the $\mathrm{pH}$ (the initial $\mathrm{pH}$ was around 3), the solution remained a free-flowing liquid, even after a prolonged reaction time ( 3 days). However, after adjusting the $\mathrm{pH}$ to 5 , the solution almost immediately started to form a gel-like material. The reaction was allowed to proceed for 2 days to ensure complete crosslinking. The chitosan gel turned to light yellow after $1 \mathrm{~h}$ and to red after 2 days. The crosslinked product did not dissolve in water, and a near quantitate yield was obtained after washing with water. According to the elemental analysis, the degree of substitution was 0.39 .

A possible reaction mechanism for the formation of the ICC is presented in Scheme 1. In the proposed reaction, glyoxal first reacts with amines of chitosan to form imine crosslinks between the chitosan molecules. Due to the aqueous instability of the imine bond and lack of a cationic charge, glyoxal is not an ideal choice to crosslink chitosan, especially when water
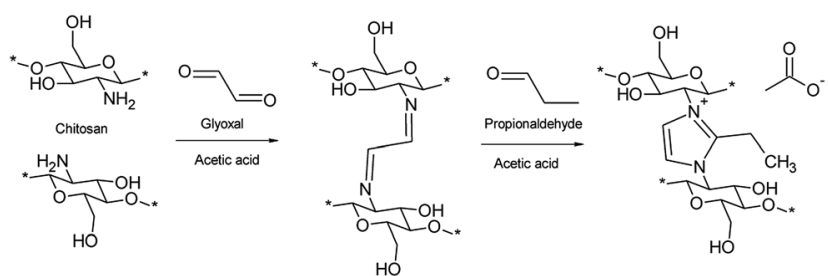

Scheme 1 Reaction mechanism illustrating the crosslinking of chitosan with Debus-Radziszewski imidazole synthesis. purification applications are concerned. The crosslinking with glyoxal is similar to that of glutaraldehyde widely used in the literature. ${ }^{12}$ The addition of aldehyde to glyoxal crosslinked chitosan leads to the formation of charged imidazole structure between the chitosan molecules. Imidazoles are known to be highly stable. Thus, the driving force underlying the crosslinking of chitosan is assumed to be the formation of imidazole moieties.

The DRIFT spectrum of chitosan shows typical stretching vibrations of $\mathrm{OH}$-groups and $\mathrm{N}-\mathrm{H}$ around $3500 \mathrm{~cm}^{-1}$ (Fig. 1). The bands at wavenumbers of 1665 and $1600 \mathrm{~cm}^{-1}$ are associated with the vibrations of the carbonyl bond $(\mathrm{C}=\mathrm{O})$ of the amide group and the vibrations of the amine group, respectively. ${ }^{23}$ In the spectrum of ICC1, aromatic $\mathrm{C}=\mathrm{C}$ stretching and $\mathrm{C}=\mathrm{N}$ ring stretching vibrations at 1581 and $1416 \mathrm{~cm}^{-1}$, respectively, are due to the formation of an imidazole group. ${ }^{24}$ The absence of a $\mathrm{C}=\mathrm{N}$ peak at $1635 \mathrm{~cm}^{-1}$ points to the absence of a significant level of glyoxal crosslinks ${ }^{25}$ and effective crosslinking of chitosan by Debus-Radziszewski imidazole synthesis.

After freeze-drying, a brownish aerogel was obtained. Despite the insolubility of ICC1, the freeze-dried aerogel was highly hydrophilic, likely due to the presence of charged groups. The aerogel absorbed up to 21 times water compared to its own mass. Based on FESEM images (Fig. S2 $\dagger$ ), the aerogel exhibited irregularly shaped macrostructure with very smooth surfaces. The ion exchange capability of the crosslinked chitosan aerogel was demonstrated by adsorbing anionic dye (Direct Yellow 27) (see Fig. S1 $\uparrow$ for the structure of the dye) with ICC1 from a model water. According to Fig. 2, the ICC1 had a high adsorption capacity (481-351 $\mathrm{mg} \mathrm{g}^{-1}$ ) in the whole studied $\mathrm{pH}$ range (2-10), and the adsorption maximum was observed at $\mathrm{pH} 4(96 \%$ retention of initial $2.5 \mathrm{mg}$ of dye by $5 \mathrm{mg}$ of ICC1). Chitosanbased materials were reported to have high retention of anionic dyes at a low pH. ${ }^{12}$ However, studies also reported sharp decreases in the adsorption capacity of chitosan-based adsorbents with alkaline solutions. ${ }^{12,26,27}$ Here, the high capacity was retained at a high $\mathrm{pH}$. This was likely due to the presence of imidazolium moieties, which have a permanent cationic charge and being more stable within a wider $\mathrm{pH}$ range than primary

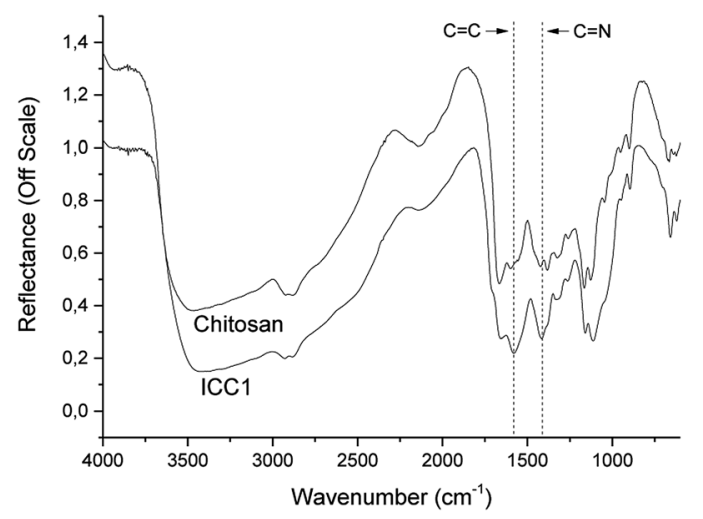

Fig. 1 DRIFT spectra of chitosan and ICC1. The $\mathrm{C}=\mathrm{C}$ stretching and $\mathrm{C}=\mathrm{N}$ ring stretching vibrations of the imidazolium moiety are marked with a dashed line. 


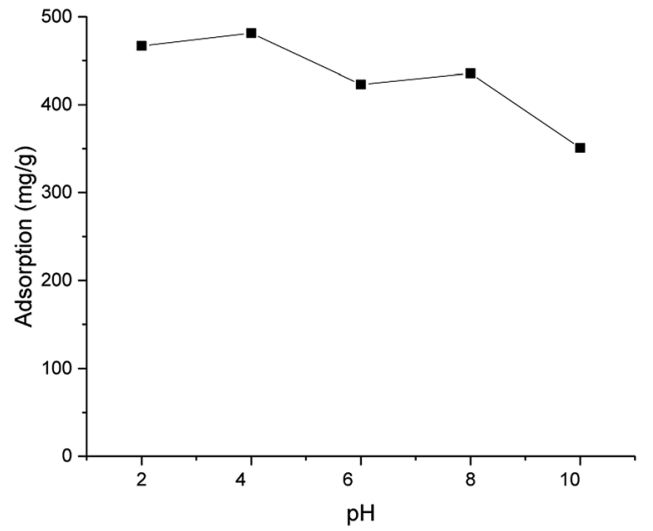

Fig. 2 Adsorption capacity of ICC1 as a function of the $\mathrm{pH}$ (conditions: $50 \mathrm{mg} \mathrm{L}^{-1}$ of dye; $100 \mathrm{mg} \mathrm{L}^{-1}$ of ICC1; 24 h shaking time; volume of 50 $\mathrm{mL}$; room temperature).

amines of chitosan. Other adsorption mechanisms, such as an aromatic interaction between the imidazolium groups of ICC, may also exist.

The results of the kinetic studies are presented in Fig. 3. The adsorption plateau was obtained after $360 \mathrm{~min}$, as only a minimal increase was observed when the shaking time was increased to $24 \mathrm{~h}$. This finding is in line with that of previous studies of chitosan-based adsorbents, where the adsorption maximum was observed after a few hours. ${ }^{27,28}$ The best fit was found using the pseudo-second order kinetic model $\left(R^{2}=\right.$ 0.99946 compared to $R^{2}=0.97242$ for the pseudo-first order kinetic model). The pseudo-second order kinetic constant was $4.35 \times 10^{-5}$. The calculated adsorption maximum was $495 \mathrm{mg}$ $\mathrm{g}^{-1}$, which is in line with the experimental value $\left(481 \mathrm{~mm} \mathrm{~g}^{-1}\right)$.

The maximum adsorption capacity was highly dependent on the initial concentration of the dye (Fig. 4). The adsorption capacity increased almost linearly from 210 to $2430 \mathrm{mg} \mathrm{g}^{-1}$ when the initial dye concentration was increased from 10 to 300 $\mathrm{mg} \mathrm{g}^{-1}$. A plateau was observed after the dye concentration increased to $300 \mathrm{mg} \mathrm{L}^{-1}$. The adsorption followed the Langmuir adsorption isotherm $\left(R^{2}=0.97352\right.$ for the Langmuir isotherm

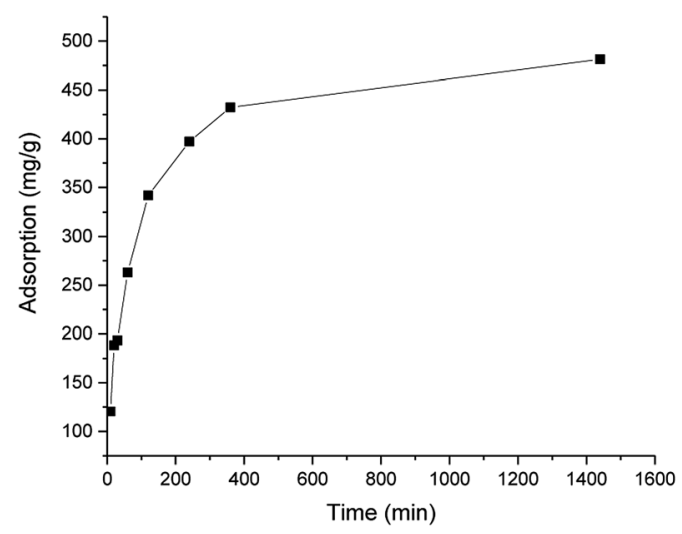

Fig. 3 Adsorption capacity of ICC1 as a function of the shaking time (conditions: $50 \mathrm{mg} \mathrm{L}^{-1}$ of dye; $100 \mathrm{mg} \mathrm{L}^{-1}$ of ICC1; total volume of 100 $\mathrm{mL}$; room temperature).

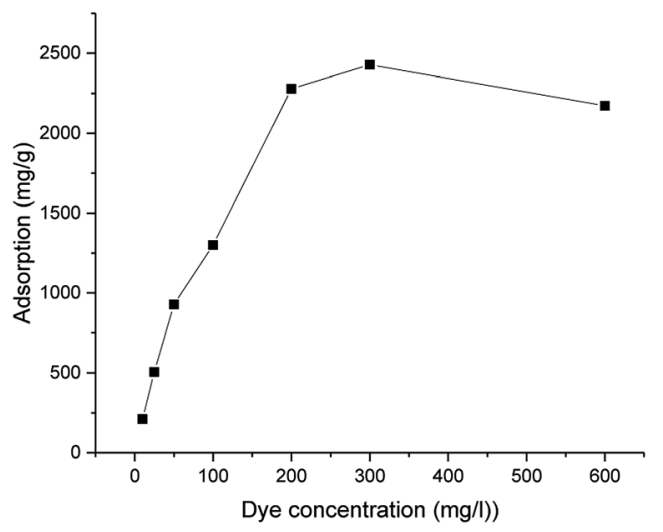

Fig. 4 Adsorption capacity of ICC1 as a function of the dye concentration (conditions: $50 \mathrm{mg} \mathrm{L}^{-1}$ of ICC1; $24 \mathrm{~h}$ shaking time; volume of 50 $\mathrm{mL}$; room temperature).

vs. $R^{2}=0.76452$ for the Freundlich isotherm). Based on the Langmuir equation (eqn (4)), the calculated adsorption maximum was $2340 \mathrm{mg} \mathrm{g}^{-1}$, which is consistent with the experimental adsorption maximum $\left(2430 \mathrm{mg} \mathrm{g}^{-1}\right)$.

The maximum adsorption capacity of ICC1 was very high compared to that of many bio-based and inorganic adsorbents. For example, the adsorption maximums of glutaraldehyde crosslinked chitosan were between 108 and $775 \mathrm{mg} \mathrm{g}^{-1}$ for 11 different anionic dyes (of note, in most cases, the adsorption capacity of glutaraldehyde-crosslinked chitosan was still higher than that of active carbon). ${ }^{29}$ Poly(acrylamide) grafted chitosan powder had adsorption maximum of $1211 \mathrm{mg} \mathrm{g}^{-1}$ towards anionic dye (Remazol Yellow Gelb 3RS). ${ }^{30}$ Of other biomacromolecule-based adsorbents, the maximum adsorption capacity of a nanocellulose-amphoteric polyvinylamine microgel was $1469.7 \mathrm{mg} \mathrm{g}^{-1}$ for an anionic dye, Acid Red GR, ${ }^{31}$ and as high as $2296 \mathrm{mg} \mathrm{g}^{-1}$ for C.I. Acid Blue 324 using tertiary amine starch ether. ${ }^{32}$ An adsorption maximum of $1800 \mathrm{mg} \mathrm{g}^{-1}$ was reported for methyl orange using inorganic $\mathrm{Mg}$-Al layered double hydroxide. ${ }^{33}$ Due to the high variety of studied dyes and adsorption experimental setups found in the literature, a direct comparison between the different adsorbents might not be meaningful (e.g., no correlation was found between the amount of charged groups in dyes and the adsorption maximum of glutaraldehyde crosslinked chitosan). ${ }^{29}$ However, it can be concluded that the anionic dye adsorption capacity of ICC1 is among the highest reported for organic and inorganic materials.

In addition to ion exchange properties, imidazole-modified chitosan can potentially be used in various other applications, such as gene delivery ${ }^{34}$ and selective separation of $\mathrm{CO}_{2}$ from $\mathrm{CH}_{4} \cdot{ }^{35}$ With these and other possible applications in mind, it would be beneficial to be able to adjust the properties of imidazole cross-linked chitosan. The feasibility of Debus-Radziszewski imidazole synthesis in chitosan crosslinking was further demonstrated by using methylglyoxal instead of glyoxal, together with propionaldehyde (ICC2). Aryl aldehyde (benzaldehyde) was also studied instead of propionaldehyde, together with glyoxal (ICC3). Methylglyoxal was also studied instead of 
glyoxal, together with propionaldehyde (ICC3). Both reactions led to the formation of ICC (DRIFT spectra and schematic illustrations of the synthesis of ICC2 and ICC3 are presented as ESI in Fig. S3 and Scheme $\mathrm{S} 1, \dagger$ respectively). The spectrum of ICC3 was similar to that of ICC. However, the wavenumber of $\mathrm{C}=\mathrm{C}$ stretching and $\mathrm{C}=\mathrm{N}$ ring stretching vibrations were higher: 1651 and $1454 \mathrm{~cm}^{-1}$, respectively. $\mathrm{C}=\mathrm{C}$ stretching and $\mathrm{C}=\mathrm{N}$ ring stretching of ICC2 occurred at 1561 and $1432 \mathrm{~cm}^{-1}$, respectively, whereas an aromatic vibration band of ICC2 was observed at a wavelength of $1615 \mathrm{~cm}^{-1}$, strongly overlapping with the amide and amine bands of chitosan. The DS of ICC2 and ICC3 was 1.51 and 1.17, respectively. The results obtained using benzaldehyde and methylglyoxal indicate that various reagents can significantly alter the reaction efficiency of chitosan crosslinking. In addition, it is possible to fabricate disparate types of ICC using divergent reagents. Thus, the properties of ICC can be adjusted in accordance with the particular application.

\section{Conclusions}

This study demonstrated that Debus-Radziszewski imidazole synthesis was an efficient method to obtain crosslinked chitosan with permanent cationic charge. A water-insoluble aerogel was obtained by a straightforward method using stoichiometric amounts of reagents. Compared to other bio-based and inorganic adsorbents, ICC showed excellent adsorption capacity of anionic dyes, indicating that the fabrication route was suitable for obtaining sustainable ion exchange material. As demonstrated here, various dicarbonyl and carbonyl compounds can be used in crosslinking chitosan with Debus-Radziszewski imidazole synthesis, thereby providing novel ways to produce chitosan-based materials with adjustable properties.

\section{Acknowledgements}

Ms Jaana Larionova is gratefully acknowledged for her contribution to the adsorption experiments. Dr Ilkka Miinalainen acknowledged for his help with the FESEM images.

\section{Notes and references}

1 A. K. Singla and M. Chawla, J. Pharm. Pharmacol., 2001, 53, 1047-1067.

2 M. N. V. Ravi Kumar, React. Funct. Polym., 2000, 46, 1-27.

3 S. Kumar, J. d. A. e Silva, M. Y. Wani, C. M. F. Dias and A. J. F. N. Sobral, J. Dispersion Sci. Technol., 2016, 37, 155-158.

4 R. C. F. Cheung, T. B. Ng, J. H. Wong and W. Y. Chan, Mar. Drugs, 2015, 13, 5156-5186.

5 P. Garg, S. Kumar, S. Pandey, H. Seonwoo, P.-H. Choung, J. Koh and J. H. Chung, J. Mater. Chem. B, 2013, 1, 6053-6065. 6 S. Kumar, J. Dutta and P. K. Dutta, Int. J. Biol. Macromol., 2009, 45, 330-337.

7 S. Kumar and J. Koh, Int. J. Mol. Sci., 2012, 13, 6102-6116.

8 S. Babel and T. A. Kurniawan, J. Hazard. Mater., 2003, 97, 219-243.
9 T. Feng and L. Xu, RSC Adv., 2013, 3, 21685-21690.

10 A. H. Gedam and R. S. Dongre, $R S C A d v ., 2015$, 5, 5418854201.

11 H. Liu and C. Wang, RSC Adv., 2013, 4, 3864-3872.

12 M. Vakili, M. Rafatullah, B. Salamatinia, A. Z. Abdullah, M. H. Ibrahim, K. B. Tan, Z. Gholami and P. Amouzgar, Carbohydr. Polym., 2014, 113, 115-130.

13 X. Shen, J. L. Shamshina, P. Berton, G. Gurau and R. D. Rogers, Green Chem., 2015, 18, 53-75.

14 H. Debus, Ann. Chem. Pharm., 1858, 107, 199-208.

15 K.-S. Krannig, D. Esposito and M. Antonietti, Macromolecules, 2014, 47, 2350-2353.

16 D. Esposito, S. Kirchhecker and M. Antonietti, Chem.-Eur. J., 2013, 19, 15097-15100.

17 M. Jiang, K. Wang, J. F. Kennedy, J. Nie, Q. Yu and G. Ma, Int. J. Biol. Macromol., 2010, 47, 696-699.

18 Z. M. dos Santos, A. L. P. F. Caroni, M. R. Pereira, D. R. da Silva and J. L. C. Fonseca, Carbohydr. Res., 2009, 344, 2591-2595.

19 S. Y. Lagergren, Zur Theorie der sogenannten Adsorption gelöster Stoffe, 1898.

20 C. Namasivayam and D. Sangeetha, Adsorption, 2006, 12, 103-117.

21 Y. S. Ho and G. McKay, Chem. Eng. J., 1998, 70, 115-124.

22 I. Langmuir, J. Am. Chem. Soc., 1918, 40, 1361-1403.

23 S. M. L. Silva, C. R. C. Braga, M. V. L. Fook, C. M. O. Raposo, L. H. Carvalho and E. L. Canedo, in Infrared Spectroscopy Materials Science, Engineering and Technology, ed. T. Theophile, InTech, 2012.

24 R. B. Sarker and A. H. Bhuiyan, Thin Solid Films, 2011, 519, 5912-5916.

25 X. Chang, D. Chen and X. Jiao, J. Phys. Chem. B, 2008, 112, 7721-7725.

26 M. M. El-Zawahry, F. Abdelghaffar, R. A. Abdelghaffar and A. G. Hassabo, Carbohydr. Polym., 2016, 136, 507-515.

27 L. Cui, Z. Xiong, Y. Guo, Y. Liu, J. Zhao, C. Zhang and P. Zhu, Carbohydr. Polym., 2015, 132, 330-337.

28 S. Chatterjee, T. Chatterjee, S.-R. Lim and S. H. Woo, Bioresour. Technol., 2011, 102, 4402-4409.

29 E. Guibal, P. McCarrick and J. M. Tobin, Sep. Sci. Technol., 2003, 38, 3049-3073.

30 G. Z. Kyzas and N. K. Lazaridis, J. Colloid Interface Sci., 2009, 331, 32-39.

31 L. Jin, Q. Sun, Q. Xu and Y. Xu, Bioresour. Technol., 2015, 197, 348-355.

32 Y. Shi, B. Ju and S. Zhang, Carbohydr. Polym., 2012, 88, 132138.

33 G. Darmograi, B. Prelot, G. Layrac, D. Tichit, G. MartinGassin, F. Salles and J. Zajac, J. Phys. Chem. C, 2015, 119, 23388-23397.

34 B. Shi, H. Zhang, Z. Shen, J. Bi and S. Dai, Polym. Chem., 2013, 4, 840-850.

35 S. Saedi, B. Nikravesh, F. Seidi, L. Moradi, A. A. Shamsabadi, M. B. Salarabadi and H. Salimi, RSC Adv., 2015, 5, 6729967307. 